\title{
A ressonância magnética no diagnóstico e estadiamento dos gliomas cerebrais: a aplicação da técnica da espectroscopia de prótons
}

\author{
The magnetic resonance imaging in the diagnosis and staging of brain \\ gliomas: the aplication of proton spectroscopy technique
}

\author{
Rodrigo Tavares Brisson', Alair Augusto Sarmet M. D. dos Santos ${ }^{2}$
}

Brisson RT, Santos AASMD. A ressonância magnética no diagnóstico e estadiamento dos gliomas cerebrais: a aplicação da técnica da espectroscopia de prótons / The magnetic resonance imaging in the diagnosis and staging of brain gliomas: the aplication of proton spectroscopy technique. Rev Med (São Paulo). 2014 jul.-set.;93(3):135-45.

RESUMO: Objetiva-se, com este trabalho, realizar uma revisão de literatura de estudos primários que abordaram a aplicação da espectroscopia de prótons por ressonância magnética $(1 \mathrm{H}-$ MRS) como método diagnóstico e de estadiamento nos gliomas cerebrais. Todos os artigos analisados, através do PubMed mostraram significância estatística na correlação entre 1H-MRS com a histopatologia da lesão. Dos 26 estudos analisados, 9 mostraram diferenças entre gliomas de outras lesões do Sistema Nervoso Central (SNC) e 23 avaliaram a diferença entre gliomas de baixo grau e gliomas de alto grau. Procurou-se mostrar que as linhas de pesquisa que usaram 1H-RMS apresentaram resultados mais refinados quanto ao diagnóstico destes tumores. Destes estudos, conclui-se que a 1H-MRS associada à MRI é um método diagnóstico com boa acurácia quando comparado com a análise histopatológica. Os metabólitos detectados pela espectroscopia se correlacionam bem com a gradação dos gliomas e fornecem fortes evidências de que este método possa ser mais utilizado para avaliação e seguimento de pacientes no pré e no pós-operatório.

DESCRITORES: Espectroscopia de ressonância magnética; Glioma/diagnóstico; Glioma/radiografia; Glioma/radioterapia; Literatura de revisão como assunto.

\begin{abstract}
The objective of this study was to perform a literature review of primary studies that assess the application of proton magnetic ressonance spectroscopy (1H-MRS) as a method in the diagnosis and staging of brain gliomas. We used the "PubMed's database" for selection of primary studies that used 1H-MRS in the diagnosis and/or staging of gliomas. All articles analyzed through PubMed's database showed statistical significance in the correlation between 1H-MRS and histopathology of the lesion. Of the 26 studies examined nine showed difference between gliomas and other lesions of the Central Nervous System (CNS) and 23 evaluated the difference between low-grade gliomas and high-grade gliomas. Sought to show that the lines of research that have used $1 \mathrm{H}-\mathrm{RMS}$ presented more refined results as to the diagnosis of these tumors. These studies have concluded that $1 \mathrm{H}-\mathrm{MRS}$ associated with MRI is a diagnostic method with good accuracy when compared with histopathological analysis. Metabolites detected by spectroscopy correlate well with the grading of gliomas and provide strong evidence that this method can be used for evaluation and followup of patients preoperatively and postoperatively.
\end{abstract}

KEYWORDS: Magnetic resonance spectroscopy; Glioma/ diagnosis; Glioma/radiography; Glioma/radiotherapy; Review literature as topic.

1. Médico graduado pela Faculdade de Medicina da Universidade Federal Fluminense (UFF) - Departamento de Radiologia; Médico Residente de Neurologia do Hospital de Clínicas da Universidade Federal do Triângulo Mineiro - UFTM, Uberaba, MG.

2. Professor e Chefe do Departamento de Radiologia da Faculdade de Medicina da Universidade Federal Fluminense-UFF.

Endereço para correspondência: Departamento de Neurologia do Hospital de Clínicas da Universidade Federal do Triângulo Mineiro. Rua Getúlio Guaritá, 330. Nossa Senhora da Abadia, Uberaba, MG, Brasil. CEP: 38025-440. E-mail: quimicarod@hotmail.com 


\section{INTRODUÇÃO}

\section{Epidemiologia}

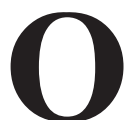

s gliomas cerebrais são a neoplasia primária do sistema nervoso central (SNC) mais prevalente, e correspondem a mais de $70 \%$ dos tumores cerebrais. Destes, os glioblastomas multiformes (GBM) são considerados o tipo histológico mais frequente e de pior prognóstico ${ }^{1}$. Os tumores gliais, de modo geral, são classificados de acordo com a classificação histológica dos tumores cerebrais primários constante na $4^{\text {a }}$ edição da Classificação de Tumores da Organização Mundial de Saúde em 2007 (Tabela 1).

Tabela 1- Classificação dos tumores cerebrais primários segundo a OMS

\begin{tabular}{lll}
\hline Tumores neuroepiteliais & Tumores das meninges & Linfomas e tumores hematopoiéticos \\
\hline - Tumores astrocitários & $\bullet$ Meningioma grau I & $\bullet$ Linfoma maligno (alto e baixo grau) \\
- Astrocitoma pilocítico (grau I) & $\bullet$ Meningioma atípico (grau II) \\
- Astrocitoma subependimário de células & $\bullet$ Meningioma anaplásico (grau III) \\
gigantes (grau I) & \\
- Astrocitoma difuso (grau II) & \\
- Xantoastrocitoma pleomórfico (grau II) & \\
- Astrocitoma anaplásico (grau III) \\
- Glioblastma (grau IV) \\
- Tumores oligodendrogliais \\
- Oligodendroglioma (grau II) \\
- Oligodendroglioma anaplásico (grau III) \\
- Tumores oligoastrocitários \\
- Oligoastrocitoma (grau I) \\
- Oligoastrocitoma anaplásico (grau III)
\end{tabular}

\section{Localização anatômica dos tumores}

A Localização é um fator importante, se não, o de maior prognóstico nos gliomas cerebrais. Os GBMs, por exemplo, são geralmente encontrados na substância branca da região supratentorial e no centro semioval ${ }^{2,3}$. São encontrados em todas as idades, sendo mais comum na idade adulta com pico de incidência entre 65-75 anos. É descrito como discretamente mais comum em homens e em indivíduos brancos. Dependendo da área cerebral acometida pelo tumor, o paciente apresenta clínica $\operatorname{distinta}^{1,2}$.

\section{Disseminação dos gliomas}

A disseminação dos gliomas ocorre mais comumente por extensão local e através do líquido cérebro-espinhal. A via hematogênica é muito rara e pode ocorrer geralmente em pacientes que sofreram cirurgias. A disseminação via linfática inexiste no SNC. A morbidade e mortalidade dos gliomas estão, portanto, relacionados ao crescimento local e extensão direta do tumor do sítio de origem no cérebro ${ }^{2}$.

\section{Etiologia e metabólitos do tumor}

A proliferação celular anormal, migração, angiogênese e ausência de apoptose estão entre os processos biológicos que determinam o caráter agressivo e a grande dificuldade de tratamento dos gliomas. Os eventos moleculares seriam responsáveis pela promoção e progressão da malignidade tumoral, conduzindo a fenótipos de baixa gradação ou a categorias mais elevadas. Mutação do gene supressor tumoral p53 (TP53), alterações na via de sinalização mediada pela proteína Ras, amplificação do receptor do fator de crescimento epidérmico (EGFR) dentre outras alterações moleculares, são descritas como alterações favoráveis ao aparecimento das neoplasias malignas do $\mathrm{SNC}^{4}$.

Com a progressão tumoral, é de se esperar: perda de área cerebral sadia, proliferação excessiva das células neoplásicas, aumento da área de inflamação e edema, aumento da neovascularização e da área de necrose.

Alguns metabólitos são encontrados no tecido cerebral normal e que, contudo, estão alterados no tecido cerebral neoplásico. São classicamente identificados os metabólitos da colina (Cho-m), N-acetilaspartato (NAA), Creatina (Cr) dentre outros metabólitos. Cho-m são compostos de colina, fosfocolina e glicerofosfocolina e são descritos como marcadores do aumento do turnover de síntese de membrana celular ou aumento da densidade celular. O NAA é descrito como um marcador neuronal contido em neurônios sadios em geral. E a $\mathrm{Cr}$ é o 
metabólito relacionado com o aumento do metabolismo celular ${ }^{5}$.

Assim, no processo de progressão tumoral é de se esperar que haja um aumento das concentrações de Cho-m e uma diminuição NAA e Cr. Entende-se, portanto, que pelo processo de proliferação desordenado, há aumento do turnover de membrana celular justificando o aumento do pico de Cho-m. A diminuição de NAA poderia ser explicada pela perda das características de diferenciação das células nervosas em produzirem este metabólito fisiologicamente. Já a diminuição do pico de $\mathrm{Cr}$ poderia ser entendida pelo processo de necrose e perda de células com metabolismo fisiológico.

Estudos têm relatado aumento dos níveis de Cho-m e diminuição dos níveis de NAA e Cr detectados utilizando-se a técnica de espectroscopia de prótons por $\mathrm{RM}^{5,6}$.

\section{Gradação dos gliomas}

Com base no potencial de infiltração e progressão para formas mais malignas, os tumores de células da glia compreendem duas grandes classes. Na primeira classe estão incluídos os astrocitomas difusos, caracterizados por uma grande capacidade de invasão, infiltração difusa de potencial para progressão tumoral. Esses tumores são classificados de acordo com a Organização Mundial de Saúde (OMS) como astrocitoma pilocítico (grau II), astrocitoma anaplásico $\{(\mathrm{AA})$ (grau III) $\}$ e glioblastoma multiforme $\{(\mathrm{GBM})$ (grau IV $)\}^{7}$. Os critérios para separação entre os graus II e III incluem o aumento da celularidade e atipia nuclear, sendo os de grau IV, distinguidos pelo aumento da celularidade, proliferação endotelial e necrose $\mathrm{e}^{3,7}$.

A outra classe é composta de tumores que tem um potencial de invasão mais limitado, comportamento menos agressivo e uma capacidade reduzida para transformação maligna. Nesta classe estão incluídos: astrocitoma pilocítico juvenil (OMS grau I), xantoastrocitoma pleomórfico e astrocitoma subependimário de células gigantes. Dependendo do grau de anaplasia, outras três variações histológicas (astrocitomas fibrilar, gemistocítico e protoplasmático) são reconhecidas e não classificadas. As características histológicas, e o comportamento pouco agressivo destes tumores impedem sua classificação no grupo dos gliomas infiltrativos. Outros tumores cerebrais primários são: os oligodendrogliomas, oligodendrogliomas mistos e ependimomas, dentre outras entidades tumorais incluídas pela WHO em 2007. Os astrocitomas e os oligodendrogliomas são freqüentemente considerados gliomas difusos, devido ao seu crescimento infiltrativo $0^{3,7,8}$

O sistema de St Anne-Mayo fornece uma classificação dos astrocitomas com uma perspectiva de estabelecer prognóstico. Esse sistema é baseado na presença ou ausência de quatro critérios: I) atipia nuclear, II) mitose, III) proliferação endotelial, IV) necrose. A classificação tumoral é determinada pelo número desses critérios: 0 critério: grau I; 1 critério: grau II; 2 critérios: grau III; 3 ou 4 critérios: grau IV. Utilizando-se esta classificação, a sobrevida média é inferior a 12 meses para o grau IV; 18 meses para o grau III; 4 anos para o grau II e média de 8 anos para o grau I. Contudo os avanços na biologia molecular permitem descrição precisa de anomalias e mutações genéticas possibilitando subsídios para um sistema de classificação mais preciso ${ }^{9,10}$.

\section{Evolução e progressão dos tumores}

Estudos sugerem que a progressão tumoral é proporcional ao período de acometimento. Essa progressão é inicialmente focal, e o diagnóstico é baseado na parte mais maligna do tumor ${ }^{11}$.

O GBM é tipicamente heterogêneo, com área central de necrose circundado por parede sólida de tecido neoplásico. Ao redor deste tecido sólido encontramos área de edema vasogênico "em dedo de luva" e que geralmente cursa com importante efeito de massa ${ }^{2}$.

Um conceito importante para entender as características patológicas dos gliomas de alto grau é que gliomas infiltrativos, isto é, graus II a IV, freqüentemente contem múltiplas áreas de variação histológica. Este conceito é importante por várias razões. A primeira delas a se considerar são os erros na coleta de área limitada na biópsia, o que poderia levar o neuropatologista a classificar uma área restrita do tumor em um grau que não corresponderia ao verdadeiro grau contido em outras áreas do tumor ${ }^{2,11}$.

Outra importante conseqüência das variações histológicas vistas em um mesmo tumor é a pobre correlação entre a radiologia convencional e a histopatologia das margens da lesão infiltrativa. Um glioma é visto nas imagens radiológicas porque produz efeito de massa, edema, necrose e hemorragia. Estas características imageológicas se correlacionam bem com as mudanças histopatológicas vistas nos tumores de alto grau, como rápido crescimento celular e neovascularização, porém não deixam claro as delimitações da margem do tumor e o possível grau ${ }^{2}$. Estudos de RM mais modernos, como a espectroscopia, a difusão, dentre outras técnicas, tem melhorado a acurácia na avaliação diagnóstica das áreas do tumor, que é o objetivo deste trabalho ${ }^{12}$.

\section{Diagnóstico}

O exame padrão ouro para o diagnóstico dos gliomas é a biópsia da lesão com análise histopatológica do material $^{13}$. Este exame oferece, como vantagem, acurácia diagnóstica, se realizada associada a técnica estereotáxica e a exames de imagem como a RM. Além disso, é considerado um exame minimamente invasivo. Como 
desvantagens, são citadas como sendo um procedimento invasivo, podendo aumentar a morbidade e mortalidade nos pacientes; Pode oferecer diagnóstico impreciso se a biópsia for dirigida a uma região não representativa do tumor; Além disso, muitos pacientes que necessitam de diagnóstico, muitas vezes já apresentam necessidade de craniotomia para redução do efeito de massa provocado pelo tumor, e assim, a possibilidade de ressecção deve ser considerada como tratamento inicial e ao mesmo tempo como método diagnóstico ${ }^{14}$.

Os exames de imagem têm adquirido importância crescente no processo de diagnóstico dos tumores intracranianos. As imagens de Ressonância Magnética (RM), em particular, emergiram na última década como uma modalidade de exame que frequentemente é usado para avaliar tumores cerebrais e tem sofrido grandes evoluções neste sentido. A RM tem participado ativamente no processo de diagnóstico, classificação, plano terapêutico e na vigilância pós-tratamento dos tumores cerebrais ${ }^{16}$. Por essa importância nas várias etapas do desenvolvimento tumoral e tratamento, tem se desenvolvido técnicas avançadas, em RM, para oferecer informações anatômicas mais precisas, e mais que isso, oferecer informações fisiológicas como a composição química de determinadas áreas ${ }^{6,15,16}$.

Além das técnicas de RM que fornecem imagens anatômicas, novas técnicas usadas na RM vão além, e destacam-se as técnicas fisiológicas de perfusão, de difusão, da espectroscopia de prótons, do nível de oxigênio sanguíneo, dentre outras ${ }^{3}$. Essas técnicas têm como vantagens serem não invasivas, porém ainda carece de estudos científicos para compararem sua sensibilidade e especificidade em relação ao exame padrão ouro: a biópsia com análise histopatológica. Além disso, pode oferecer para o neurocirurgião a melhor área para biópsia e evitar fragmentos de tecido não representativos do tumor não vistas macroscopicamente no peri-operatório, aumentando assim a acurácia do estadiamento ${ }^{6,12,14}$.

\section{Tratamento}

Otratamentodosgliomas malignos, particularmente dos GBM, requer uma abordagem multidisciplinar considerando-se a natureza incurável desta doença. $\mathrm{O}$ tratamento preconizado até o momento inclui: cirurgia, radioterapia e quimioterapia ${ }^{17}$. A excisão cirúrgica total ou parcial continua sendo a principal abordagem terapêutica contra os tumores primários do SNC. A máxima extirpação do tecido tumoral o mais precoce possível pode influenciar na sobrevida do paciente. Outros benefícios do tratamento cirúrgico consistem na probabilidade de um acurado diagnóstico histológico, na melhora dos déficits instalados, e na redução dos riscos de progressão tumoral. Contudo, o potencial infiltrativo da maioria dos gliomas, assim como a capacidade de migração das células tumorais, pode tornar ineficaz o tratamento cirúrgico ${ }^{14,17,18,19}$. Nos pacientes portadores de gliomas de baixo grau, uma proporção significativa é tratada de modo conservador, com drogas anti-convulsivantes acompanhados por exames de imagem ${ }^{18}$.

As estratégias terapêuticas alvo-direcionadas prometem significativos avanços no tratamento desses tumores através do desenvolvimento de agentes mais específicos, com menor grau de toxicidade e induzindo a apoptose, sendo, portanto, mais efetivos ${ }^{18}$. Dentro desta perspectiva, o monitoramento do tumor nos casos de tratamento conservador com a espectroscopia de prótons já mostrou resultados positivos em alguns trabalhos ${ }^{20,21,22}$.

\section{JUSTIFICATIVA DO ESTUDO}

A biópsia, por ser um método invasivo, não está livre de complicações durante e após o procedimento e nem de erros diagnósticos e de estadiamento ${ }^{14}$. A busca de exames alternativos menos invasivos, com sensibilidade e especificidade semelhante ao da biópsia com análise histopatológica, considerada padrão ouro para o diagnóstico e estadiamento dos gliomas, vem ganhando destaque em pesquisas recentes ${ }^{18}$.

As imagens da ressonância magnética convencionais fornecem detalhes da anatomia cerebral além de dados importantes para o diagnóstico das lesões cerebrais, incluindo-se ai os tumores cerebrais ${ }^{23}$. A técnica da espectroscopia de prótons $\left({ }^{1} \mathrm{H}\right)$ por ressonância magnética do cérebro permite identificar, in vivo e de modo näo-invasivo, neurometabólitos pertencentes a diversas vias do metabolismo intermediário ${ }^{24}$.

A espectroscopia de hidrogênio, clinicamente falando, é a mais utilizada por ser, o hidrogênio, o elemento químico mais abundante no organismo. É encontrado em tecidos normais e anormais, em quantidades diferentes. Esses prótons estão contidos em substancias no cérebro denominados neurometabólitos. Os neurometabólitos que podem ser identificados pela espectroscopia (respeitando o limite inferior de concentração para sua detecção, cerca de 0,5 e $1,0 \mathrm{mmol} / 1$ são os já citados NAA, Cho-m, Cr, lipídios, dentre outros ${ }^{25}$. É importante saber que esses metabólitos variam de acordo com a região cerebral analisada, a idade do paciente e a presença de patologia na região $0^{26}$. Assim, ao invés de imagens anatômicas, a espectroscopia de prótons por RM apresenta-se na forma de gráficos que demonstram picos destes metabólitos que apresentam diferentes radiofreqüências e intensidades ${ }^{24}$.

Alguns autores já relatam o uso da espectroscopia para diferenciação entre lesão focal neoplásica e nãoneoplásica, diferenciação de radionecrose e recidiva tumoral, e como instrumento de caracterização de doenças degenerativas ${ }^{26,27,29}$. Diversos estudos vêm mostrando o advento da espectroscopia no diagnóstico diferencial 
das massas cerebrais ${ }^{29}$. Ainda não está claro o papel da espectroscopia de prótons no diagnóstico e estadiamento dos gliomas cerebrais em função do pequeno número de pesquisas neste sentido. Contudo, estudos já tem mostrado o potencial desta técnica no diagnóstico e estadiamento dos tumores cerebrais ${ }^{5,30,31}$.

Sendo assim, este trabalho visa saber se a técnica da espectroscopia de prótons por ressonância magnética tem boa sensibilidade e especificidade no diagnóstico e estadiamento dos gliomas cerebrais, quando comparados com a técnica padrão ouro: a biópsia com análise histopatológica.

\section{OBJETIVOS}

Objetiva-se, com este trabalho, realizar uma revisão de literatura de estudos primários que abordaram a aplicação da 1H-RM- espectroscopia como método diagnóstico e de estadiamento nos gliomas cerebrais. Ao avaliar estes estudos, procurou-se saber se a espectroscopia é um exame com sensibilidade e especificidade adequadas quando comparado com a análise histopatológica da lesão.

\section{MATERIAIS E MÉTODOS}

Foi utilizado a base de dados do "PubMed" para seleção de estudos primários que utilizaram a espectroscopia de prótons por RM no diagnóstico e/ou estadiamento dos gliomas. Como critérios utilizados para selecionar os artigos para a revisão sistemática após a busca foram:

1. Ser estudo primário;

2. Utilizar a espectroscopia de prótons por RM como exame principal a ser comparado com a análise histopatológica dos gliomas (padrão ouro);

3. Os estudos devem estar focados no estudo dos gliomas e tumores intra-axias metastáticos (estes últimos desde que façam diagnóstico diferencial com os gliomas pela técnica da espectroscopia);

4. A correlação histopatológica obedece aos critérios da OMS;

5. Correlacionar através do valor de $p$ se há ou não diferença estatisticamente significativa entre os dados analisados, sendo considerado significativo se $\mathrm{p}<0.05$.

Como critérios de exclusão dos estudos para compor a revisão sistemática foram considerados:

1. Estudos que não fossem primários;

2. Estudos que não utilizaram a espectroscopia como método diagnóstico ou como método de estadiamento nos tumores cerebrais;
3. Estudos que não tem foco nos gliomas cerebrais;

4. Estudos que não fazem correlação com a histopatologia em graus estabelecidos pela OMS;

5. Estudos que comparam o uso da espectroscopia com outro exame que não a histopatologia;

6. Estudos que não utilizaram os metabólitos NAA, Cho-m, Cr e lipídios como um dos pontos centrais do experimento;

7. Possui amostra menor do que 10 pacientes.

Foram utilizadas as seguintes palavras-chave na barra de busca: "glioma", "mr", "espectroscopy", "diagnostic" e "grading”. Estas palavras foram digitadas em minúsculo e nesta ordem. Não foi selecionado nenhum outro parâmetro para a seleção dos artigos como data da publicação ou período de interesse. Foram considerados todos os artigos desde a data da pesquisa (30/12/2011) para datas anteriores de publicação.

Nesta busca foram encontrados 54 artigos. Destes, respeitando os critérios de inclusão e exclusão, foram selecionados 26 para compor este estudo de revisão.

\section{RESULTADOS}

Dos artigos analisados, todos mostraram, em suas avaliações, significância estatística na associação entre a capacidade de se fazer o diagnóstico de gliomas cerebrais quando comparado com a histopatologia da lesão. Dos 26 estudos analisados, nove foram capazes de diferenciar gliomas de outras lesões do SNC. Vinte e três estudos foram capazes de avaliar a diferença entre gliomas de baixo grau e gliomas de alto grau. Dois estudos foram capazes de diferenciar gliomas de metástases. Em todos os estudos analisados houve tendência em mostrar o benefício do uso da espectroscopia para guiar a melhor área da biopsia, estabelecer meios diagnostico alternativos além da biopsia e possível acompanhamento dos pacientes no pós-operatório.

Observando os artigos analisados e organizando-os pelas datas de publicação, das mais antigas para as mais atuais, procurou-se mostrar como se evoluiu as linhas de pesquisa desde os primeiros trabalhos até os mais recentes utilizando a espectroscopia de prótons por RM (1H-RMS) e os seus respectivos resultados.

Estudos in vitro realizados por Kinoshita et al. ${ }^{32}$ em 1993, foram capazes de mostrar aumento significativo dos níveis de Cho-m e diminuição nos níveis de NAA e $\mathrm{Cr}$ em extratos de tumores cerebrais primários quando comparados com extratos de cérebros normais $(p<$ $0.05)$. Além disso, o estudo ainda faz menção a possível capacidade de estadiamento tumoral usando-se esses metabólitos.

Poptani et al..$^{29}$, com uma amostra de 120 pacientes com massas intracranianas, avaliou a capacidade da espectroscopia fazer o diagnóstico diferencial entre essas lesões e os gliomas cerebrais. Todos os gliomas 
de alto e baixo grau mostraram altas concentrações de Cho-m e baixas concentrações de NAA e Cr. Contudo, a razão de NAA/Cho-m foi significativamente menor e a razão Cho-m/Cr maior em gliomas de alto grau quando comparado aos gliomas de baixo grau. A presença de picos de lipídios sugere alto grau de malignidade, sendo achado mais comum em metástases e GBM. Todas as metástases mostraram altas concentrações de lipídios e lactato mas não de Cho-m. Abscessos piogênicos mostraram altas concentrações de aminoácidos, lactato, alanina e acetato. Tuberculomas mostraram apenas picos de lipídios. Todas as associações tiveram $p<0.05$, exceto a razão de NAA/ $\mathrm{Cr}$, que não mostrou diferença entre gliomas de alto e baixo grau.

Carpinelli et al. ${ }^{33}$, com uma amostra de 36 pacientes, mostrou em suas analises que há diferença estatisticamente significativa entre astrocitomas e GBM considerando-se as razões de Cho-m/Cr. O estudo também aponta para outros metabólitos como a glicina e o NAA como metabólitos importantes na diferenciação de tumores.

Carapella et al. ${ }^{34}$, mostrou que a espectroscopia é capaz de diferenciar astrocitomas de GBM $(\mathrm{p}<0.002)$ a partir dos níveis de Cho-m e Cr.

Zakrzewski et al. ${ }^{35}$, com uma amostra de 25 crianças com tumores de fossa posterior, mostrou que a concentração de Cho-m e lactato, e a razão Cho/NAA foram os parâmetros de maior significância estatística para se diferenciar tumores de alto grau dos de baixo grau especialmente entre astrocitoma pilocítico e meduloblastoma.

Kaminogo et al.$^{36}$, em uma amostra de 25 pacientes com diagnostico de glioma mostrou que a razão NAA/ Cho-m foi significativamente menor nos tecidos tumorais do que no tecido cerebral sadio $(p<0.0001)$, porém não mostrou significância entre os graus do tumor. A razão entre $\mathrm{Cr} / \mathrm{Cho}-\mathrm{m}$ teve diferença significativa entre todos os gliomas e os tecidos sadios $(p<0.0001)$ e entre os gliomas graus II e IV $(p<0.0005)$.

Fountas et al. $^{37}$, ao estudar a aplicação da espectroscopia no estudo dos gliomas em 120 pacientes, concluiu que os gliomas foram caracterizados por um aumento da concentração de Cho-m e diminuição de NAA e Cr. Subsequentemente neste mesmo estudo observou-se aumento na razão $\mathrm{Cho} / \mathrm{Cr}$. Foi encontrado altas concentrações de lipídios em gliomas de alto grau. E comparando a espectroscopia com o estudo histopatológico, este exame acertou o diagnóstico de glioma em 85,6\% dos casos. Além disso, foi capaz de estadiar corretamente o tumor em alto ou baixo grau em $86,3 \%$ dos casos. Os abscessos puderam ser diferenciados dos tumores pela ausência de Cho-m, Cr e NAA e pela presença de lipídios, lactato e aminoácidos. Em 2004, este mesmo autor ${ }^{38}$, agora com 71 pacientes, quantifica melhor seus estudos e revela que além do aumento de Cho-m, há diminuição das concentrações de Cr e NAA nos gliomas. Outros metabolitos variam inconsistentemente mesmo entre tumores de mesmo grau. Além disso, conclui que a razão $\mathrm{Cho/Cr}$ é um marcador muito importante no estadiamento dos tumores em alto ou baixo grau $(p<$ 0.043). A razão dos outros metabólitos não mostraram significância estatística nas associações.

Yang et al. ${ }^{39}$, estudou em 17 pacientes com gliomas histologicamente confirmados a aplicação da $1 \mathrm{H}-$ RMS. A média da razão máxima de Cho-m/NAA, Cho/ $\mathrm{Cr}$ e da razão mínima de NAA/Cr foram estatisticamente significativas $(p<0.05)$ quando comparamos gliomas de alto e baixo graus.

Möller-Hartmann et al..$^{40}$, em um estudo com 176 pacientes, mostrou a capacidade da espectroscopia em diferenciar gliomas de outras massas não neoplásicas. De seus estudos pôde-se concluir que áreas de lesões não neoplásicas como áreas cerebrais de infarto e abscessos são marcadas por diminuição nos níveis de Cho-m, $\mathrm{Cr}$ e NAA, enquanto tumores geralmente mostram elevados níveis de Cho-m e baixos níveis de Cr e NAA. Quanto mais maligno o tumor primário, maiores são os níveis de Cho-m e Lipídios. As metástases podem ser diferenciadas dos gliomas de alto grau pelos níveis de lipídios ainda maiores neste caso. Nos tumores extra-axiais observase ausência de NAA (marcador neuronal). A capacidade de diagnóstico correto com a espectroscopia associada à RM convencional foi de $70.5 \%$. O estudo informa que as diferenças na comparação entre os metabólitos são estatisticamente significativa com $\mathrm{p}<0.05$.

Bulakbasi et al..$^{41}$, concluiu em seu estudo com 49 pacientes que, tumores malignos podem ser facilmente diferenciados de tumores benignos pela diminuição mais acentuada da razão de NAA/Cho e NAA/Cr e razões mais altos de lactato/lipídio e lactato/Cr nos tumores malignos em relação aos tumores benignos $(p<0.05)$. O estudo ainda cita que é possível diferenciar metástases de tumores primários de alto grau, pois a razão de lipídio/ $\mathrm{Cr}$ é maior nas metástases $(p<0.05)$. Os meningiomas podem ser distinguidos dos outros gliomas pela alta razão de Alanina/Cr.

Law et al. ${ }^{42}$, em estudo com 160 pacientes mostrou que a espectroscopia tem boa acurácia para o estadiamento dos gliomas cerebrais. Houve diferenças estatisticamente significativas entre as razoes de Cho-m/Cr, Cho/NAA e $\mathrm{NAA} / \mathrm{Cr}$ entre gliomas de alto e baixo grau, com valor de $p$ respectivamente menores que $0.012,0.001$ e 0.0038 .

Murphy et al. ${ }^{43}$, mostrou em uma amostra de 20 pacientes que todos os tumores de alto grau tiveram maiores níveis de lipídios (figura 4), quando comparados aos tumores de baixo grau $(p<0.001)$.

Magalhães et al. ${ }^{44}, 2005$, em estudo com 27 pacientes foram capazes de mostrar que gliomas de alto grau possuem alto grau da razão Cho-m/NAA e Cho-m/ 
Cr quando comparados com gliomas de baixo grau (figura 5 e 6). Contudo, associação estatística não foi observada no estudo.

Chen et al. ${ }^{45}$, em seu estudo com 34 pacientes investigou a relação entre as alterações vistas à espectroscopia e o potencial proliferativo dos tumores astrocíticos. Seus resultados mostraram que as razões de Cho-m/NAA e de Cho-m/Cr nos astrocitomas de alto grau foram significativamente maiores do que os de baixo grau $(p<0.009 ; p<0.001)$.

$\mathrm{Xu}$ et al. ${ }^{46}$, com uma amostra de 25 pacientes mostrou que a espectroscopia pode diferenciar tumores de alto grau de tumores de baixo graus quantificando Cho-m. De modo que a mensuração de Cho-m em oligodendrogliomas de alto grau foi maior que nos de baixo grau $(p<0.001)$, assim como a presença de lipídios e lactato estão maiores em tumores de alto grau $(p<$ 0.014). A acurácia para graduar oligodendrogliomas foi de $90 \%$ para Cho-m e $85 \%$ para lactato ou lipídios.

Stadlbauer et al. ${ }^{5}$, com uma amostra de 26 pacientes avaliou o uso da espectroscopia para estadiar gliomas cerebrais utilizando os metabólitos Cho-m, NAA e $\mathrm{Cr}$ e suas razões. Foram encontrados baixos níveis de Cho-m e altos níveis de NAA em tumores de graus II quando comparados com tumores grau III $(p<0.01)$. A razão Cho-m/NAA no centro do tumor mostrou diferença estatisticamente significativa entre tumores grau II e grau III $(p<0.001)$. O estudo mostrou ainda correlação entre a evolução histopatológica da lesão e as mudanças na concentração molar dos metabólitos, mostrando associação linear negativa para o NAA com a progressão da infiltração do tumor e positiva para o Cho-m $(p<$ 0.001).

Toyooka et al. ${ }^{47}$, em 2008 com uma amostra de 33 pacientes mostrou que a média da razão $\mathrm{Cho/Cr}$ foi útil para discriminar tumores de grau II e III e a média da razão Lactato/Cr foi útil para discriminar Grau III de Grau IV. A razão de Cho-m/Cr foi estatisticamente significativa para diferenciar graus II, III e IV $(p<0.01)$. Um valor também significativo foi encontrado na diferenciação entre graus II, III e IV $(p<0.01)$. Outras associações não tiveram significância estatística.

Chawla et al. ${ }^{48}$, mostrou, in vivo, que em áreas do glioma com hiperfluxo apresenta-se com elevações do pico de Cho-m em relação a substancia branca do lado normal do cérebro $(p<0.05)$. E esse pico é maior nas áreas de hiperfluxo de tumores de alto grau, quando comparado ao de tumores de baixo grau.

Zhou et al. ${ }^{49}$, em uma amostra de 36 pacientes, mostrou que houve diferença significativa nas razões de NAA/Cr, Cho-m/Cr, e NAA/Cho entre gliomas de baixo grau e o tecido cerebral contralateral normal, e entre gliomas de alto grau e o tecido cerebral normal contralateral $(p<0.01)$. Os gliomas de de baixo grau e os de alto grau diferiram significativamente nas razões de $\mathrm{NAA} / \mathrm{Cr}$, Cho-m/Cr e NAA/Cho $(p<0.05)$ e entre a área peritumoral sem edema e o tecido do glioma $(p<0.05)$. Entre área peritumoral sem edema e a área contralateral cerebral sadia, a razão de NAA/Cr e NAA/Cho-m foram significativamente diferentes $(p<0.05)$, mas a razão do Cho-m/Cr não teve significância estatística.

Senft et al. ${ }^{50}$, com uma amostra de 63 pacientes, utilizou a espectroscopia para caracterizar os gliomas cerebrais. Os resultados mostraram que houve diferença estatisticamente significativa entre o máximo $(p<0.05)$ e a media $(p<0.0001)$ dos valores de Cho-m entre os gliomas graus II e III/IV. E a acurácia diagnóstica do total Máximo de Cho-m (82,5\%) foi maior que a razão $\mathrm{Cho/Cr}$ $(72,1 \%)$ e a média de Cho-m $(71,4 \%)$.

Weis et al. ${ }^{51}$, com uma amostra de 20 pacientes com gliomas de diversos graus, utilizando-se a comparação dos espectros dos metabólitos NAA, Cho-m e Cr entre gliomas grau II e grau IV, mostrou que houve diferença estatisticamente significativa $(p<0.05)$ apenas para $\mathrm{Cr}$ total. Não houve diferença estatisticamente significativa entre Cho-m total e NAA total entre gliomas grau II e IV.

Frati et al..$^{52}$, em seu estudo com 296 casos, mostrou que a razão espectroscópica de Cho/NAA em diferentes áreas do mesmo tumor se correlacionam positivamente bem com a gradação histológica da lesão $(p<0.01)$, quanto maior a razão maior a malignidade.

Bendini et al. ${ }^{53}$, em um grupo de 159 pacientes, mostrou alta especificidade e sensibilidade em diferenciar GBM de metástases com espectroscopia associada a MRI $(\mathrm{p}<0.000001)$. Este mesmo método, utilizando a razão Cho/Cr e pico de lipídeos, foi capaz de distinguir gliomas graus III-IV de gliomas graus II.

Mais recentemente, Zeng et al. ${ }^{54}$, em 2011, com 39 pacientes, mostrou que as razões de Cho-m/Cr e Cho-m/ NAA foi significativamente maior em tumores de alto grau do que em tumores de baixo grau $(p<0.001)$.

Por último, Zou et al. ${ }^{55}$, no fim de 2011, realizaram estudo com uma amostra de 30 pacientes, e encontraram diferença estatisticamente significativa entre gliomas de alto e baixo graus para as razões de Cho-m/Cr, NAA/Cr e NAA/Cho $(p<0.01)$. Fortalecendo o uso da 1H-MRS como alternativa no diagnóstico diferencial e estadiamento dos gliomas cerebrais.

\section{DISCUSSÃO}

Foi realizada uma revisão de literatura estudos primários que utilizaram a Espectroscopia de Prótons por RM (1H-MRS) para diagnóstico e estadiamento dos gliomas cerebrais, buscando saber como a literatura tem abordado o tema nos últimos anos. Para o levantamento de informações, não foram estabelecidos limites de data e optou-se por analisar sistematicamente os trabalhos por 
Brisson RT, et al. A ressonância magnética no diagnóstico e estadiamento dos gliomas cerebrais.

ordem cronológica de publicação.

A maioria dos estudos que mostraram resultados consistentes foi publicada nos últimos 10 anos. Muito provavelmente, artigos que não revelaram associações estatisticamente significativas não foram publicados, e, por isso, este estudo não está livre de viés de seleção. Apenas um estudo analisado ${ }^{51}$ não mostrou associação estatisticamente significativa na diferenciação entre tumores de alto e baixo grau ao utilizar a 1H-MRS na análise quantitativa de metabólitos classicamente descritos (Cho-m e NAA). Contudo, a análise destes artigos, de certa forma, forneceu evidencia significativa do valor da 1H-MRS na avaliação dos gliomas. Com isso, espera-se estimular a realização de mais estudos primários, com amostras maiores e multicêntricos.

$\mathrm{Na}$ maioria dos estudos não se observou "cegamento", ou seja, já se sabia o diagnóstico e o grau do tumor e sabendo-se disso foi verificado se houve ou não correspondência com a histopatologia. Os estudos foram, em sua maioria, prospectivos.

A acurácia diagnóstica da espectroscopia em combinação com MRI foi fortemente observada na maioria dos estudos. A performance da $1 \mathrm{H}-\mathrm{MRS}$ pôde ser bem analisada no estudo de Moller-Hartmann et al. ${ }^{40}$, que mostrou ser melhor quando associada a MRI.

Autilização da curva ROC, para testes diagnósticos, pelos autores foi uma estratégia importante na análise dos dados, pois fornece informações de fácil entendimento do desempenho do teste diagnóstico e leva em consideração as especificidades e sensibilidades dos testes diagnósticos. Um bom teste diagnóstico tem boa sensibilidade e especificidade e é o que os estudos tem mostrado em relação a 1H-MRS no diagnóstico e estadiamento dos gliomas cerebrais.

A maioria dos estudos analisados possuíam uma amostra com poucos pacientes, o que pode levar a erros estatísticos com validade interna e externa duvidáveis. A necessidade de estudos com amostras maiores de pacientes é requerida para que se obtenha um estudo que seja representativo da população, ou seja, possa ser extrapolado para a população.

Alguns estudos carecem de clareza no processamento dos dados, não revelando algumas vezes a associação estatística, informação esta de extrema importância para não ocorrer erros sistemáticos do tipo I ou do tipo II.

Quando analisamos os metabólitos, os que tiveram maior importância e merecem ser estudados com maior força são os Cho-m, Cr, NAA e os lipídeos. As razões destes metabólitos fornecem dados para associações mais fortes. Os metabólitos que mais foram capazes de diferenciar gliomas de alto de gliomas de baixo graus, pelos estudos analisados, foram Cho-m/Cr, Cho-m/NAA e NAA/Cr. Conforme já esclarecido anteriormente, com o processo de progressão tumoral é de se esperar que haja um aumento das concentrações de Cho-m e uma diminuição NAA e Cr. Entende-se, portanto, que pelo processo de proliferação desordenado, há aumento do "turnover" de membrana celular justificando o aumento do pico de Cho-m. A diminuição de NAA, poderia ser explicada pela perda das características de diferenciação das células nervosas que tem como marcador o NAA fisiologicamente. Já a diminuição do pico de $\mathrm{Cr}$ explicase pelo processo de necrose e perda de células com metabolismo fisiológico. Assim, também é de se esperar aumento na razão Cho-m/Cr e Cho-m/NAA e diminuição de NAA/Cr e NAA/Cho.

O impacto da espectroscopia no diagnóstico e a redução da necessidade de biópsia ainda não foi concretamente estudado, mas pelas evidências que tem mostrado, terá grande impacto na mudança de abordagem diagnóstica em pacientes com gliomas com a popularização de seu uso.

A discussão atual, em torno do uso da $1 \mathrm{H}-\mathrm{MRS}$, questiona se o uso desta técnica faz ou não a diferença no diagnóstico diferencial das massas intracranianas e no estadiamento dos gliomas cerebrais, diante de técnicas mais modernas como a técnica de perfusão, difusão, dentre outras. Deste modo, como um exame complementar, que não utiliza isótopos radioativos e é de fácil aquisição e disponibilidade clínica, a 1H-MRS pode ser usada isolada ou associada a outras técnicas, mais modernas ou não, para o diagnóstico e estadiamento de gliomas cerebrais visto sua boa acurácia como mostrada nos estudos acima.

Uma vez que auxilia o cirurgião na decisão de se intervir cirurgicamente ou não, além de servir como uma base informativa para o prognóstico do paciente, a $1 \mathrm{H}-\mathrm{MRS}$ tem seu uso na prática clínica recomendado de modo a acrescentar informações em caso de dúvidas diagnósticas e em alguns casos pode fazer a diferença na abordagem do paciente auxiliando o cirurgião a tomar uma conduta terapêutica mais segura e adequada com benefício final para o paciente.

\section{CONCLUSÃo}

Destes 26 casos analisados, pudemos observar que a 1 H-MRS associada a MRI é um método diagnóstico com boa acurácia quando comparado com a análise histopatológica. Os metabólitos detectados pela espectroscopia se correlacionam bem com a graduação do tumor e fornece fortes evidências de que este método possa ser utilizado para avaliação e seguimento de pacientes no pré e no pós operatório, associado ou não a outras técnicas, reduzindo-se assim a quantidade de biópsias a serem realizadas no seguimento destes pacientes.

A espectroscopia mostrou boa capacidade de diferenciar massas intra-axiais neoplásicas ou não. Contudo, há a necessidade de estudos maiores que possuam uma amostra mais representativa e com isso possa se fazer 
a extrapolação das utilidades da 1H-RMS para a população em geral, visto que diante de outras técnicas mais modernas, têm-se deixado de lado a utilização de uma técnica com boa sensibilidade e acurácia no diagnóstico de massas tumorais. Assim, defendemos seu uso na prática clínica de modo a complementar a certeza diagnóstica do exame de imagem, reduzindo ou mesmo evitando procedimentos invasivos

\section{REFERÊNCIAS}

1. Shandana SR, Movva S, Arora M, Singh T. Primary brain tumors in adults. Am Fam Physician. 2008;77(10):142330. Available from: http://www.aafp.org/afp/2008/0515/ p1423.html.

2. Rees JH, Smirniotopoulos JG, Jones RV, Wong K. Glioblastoma multiforme: radiologic-pathologic correlation. Radiographics. 1996;16(6):141338; quiz 1462-3. doi: http://dx.doi.org/10.1148/ radiographics.16.6.8946545

3. Braga FM, Melo PMP. Guias de medicina ambulatorial da Universidade Federal de São Paulo-UNIFESP: neurocirurgia. São Paulo: Manole; 2005.

4. da Fonseca CO, Schwartsmann G, Fischer J, Nagel J, Futuro D, Quirico-Santos T, Gattass CR. Preliminary results from a Phase I/II study of perillyl alcohol intranasal administration in adults with recurrent malignant gliomas. Surg Neurol. 2008;70(3):259-66. doi: 10.1016/j. surneu.2007.07.040.

5. Stadlbauer A, Gruber S, Nimsky C, Fahlbusch R, Hammen T, Buslei R, Tomandl B, Moser E, Ganslandt $\mathrm{O}$. Preoperative grading of gliomas by using metabolite quantification with high-spatial-resolution proton MR spectroscopic imaging. Radiology. 2006;238(3):958-69. doi: http://dx.doi.org/10.1148/radiol.2382041896.

6. Ramadan S, Andronesi OC, Stanwell P, Lin AP, Sorensen AG, Mountford CE. Use of in vivo two-dimensional MR spectroscopy to compare the biochemistry of the human brain to that of glioblastoma. Radiology. 2011;259(2):540-9. doi: 10.1148/radiol.11101123.

7. Kleihues P, Burger PC, Aldape KD, et al. Glioblastoma. In: Louis DN, Ohgaki H, Wiestler OD, editors. WHO classification of tumors of the central nervous system. Lyon: IARC; 2007.

8. Louis DN, Hiroko O, Wiestler OD, Cavenee WK, Burger PC, Jouvet A, Scheithauer BW, Kleihues P. The 2007 WHO classification of tumours of the central nervous system. Acta Neuropathol. 2007;114(2):97-109.

9. Figarella-Branger D, Bouvier C. Classification anatomopathologique des gliomes: faits et controverses. Bull Cancer. 2005;92(4):301-9. Available from: http:// goo.gl/Glc0M8.

10. Fuller CE, Perry A. Molecular diagnostics in central nervous system tumors. Adv Anat Pathol. 2005;12(4):18094. Available from: http://goo.gl/HLWCZy.

11. Collins,VP. Brain tumours: classification and genes. J rotineiros para diagnóstico e acompanhamento de massas intracranianas. Encorajamos os profissionais médicos, em particular os clínicos e cirurgiões que tem como área de atuação pacientes oncológicos, a conhecer a técnica e usála em momento oportuno em benefício diagnóstico aos nossos pacientes.

Neurol Neurosurg Psychiatry. 2004;75(Suppl II):ii2-ii11. doi: 10.1136/jnnp.2004.040337.

12. Stadlbauer A. Moser E, Gruber S, Buslei R, Nimsky C, Fahlbusch R, Ganslandt O. Improved delineation of brain tumors: an automated method for segmentation based on pathologic changes of 1H-MRSI metabolites in gliomas. Neuro Image. 2004;23(2):454-61. doi:10.1016/j. neuroimage.2004.06.022.

13. Brat DJ, Prayson RA, Ryken TC, Olson JJ. Diagnosis of malignant glioma: role of neuropathology. J Neurooncol. 2008;89:287-311. doi: 10.1007/s11060-008-9618-1.

14. Jackson RJ, Fuller GN, Abi-Said D, Lang FF, Gokaslan ZL, Shi WM, Wildrick DM, Sawaya R. Limitations of stereotatic biopsy in the initial management of gliomas. Neuro Oncol. 2001;3(3):193-200. Available from: http:// neuro-oncology.oxfordjournals.org/content/3/3/193.long.

15. Plathow C, Weber WA. Tumor cell metabolism imaging. J Nuclear Med. 2008;49(Suppl 2):43S-63S. doi: 10.2967/ jnumed.107.045930.

16. Al-Okaili RN, Krejza J, Wang S, Woo JH, Melhem ER. Advanced MR imaging techniques in the diagnosis of intraaxial brain tumors in adults. Radiographics. 2006;26(Suppl 1):S173-89. doi: http://dx.doi.org/10.1148/ rg.26si065513.

17. Gilbert MR. Recurrent glioblastoma: a fresh look at current therapies and emerging novel approaches. Semin Oncol. 2011;38(6 Suppl 4):S21-33. doi: 10.1053/j. seminoncol.2011.09.008.

18. Sanai N, Chang S, Berger MS. Low-grade gliomas in adults. J Neurosurg. 2011;115(5):948-65. doi: 10.3171/2011.7.JNS101238.

19. Storstein A, Helseth E, Johannesen TB, Schellhorn T, Mørk S, van Helvoirt R. High-grade gliomas in adults. Tidsskr Nor Laegeforen. 2011;131(3):238-41. doi: 10.4045/ tidsskr.09.1362.

20. Guillevin R, Menuel C, Duffau H, Kujas M, Capelle L, Aubert A, Taillibert S, Idbaih A, Pallud J, Demarco G, Costalat R, Hoang-Xuan K, Chiras J, Vallée JN. Proton magnetic resonance spectroscopy predicts proliferative activity in diffuse low-grade gliomas. J Neurooncol. 2008;87(2):181-7. doi: 10.1007/s11060-007-9508-y.

21. Stadlbauer A, Buchfelder M, Salomonowitz E, Ganslandt O. Fiber density mapping of gliomas: histopathologic evaluation of a diffusion-tensor imaging data processing 
Brisson RT, et al. A ressonância magnética no diagnóstico e estadiamento dos gliomas cerebrais.

method. Radiology. 2010;257(3):846-53. doi: 10.1148/ radiol.10100343.

22. Tsien C, Galbán CJ, Chenevert TL, Johnson TD, Hamstra DA, Sundgren PC, Junck L, Meyer CR, Rehemtulla A, Lawrence T, Ross BD. Parametric response map as an imaging biomarker to distinguish progression from pseudoprogression in high-grade glioma. J Clin Oncol. 2010;28(13):2293-9. doi: 10.1200/JCO.2009.25.3971.

23. Sartor K. MR imaging of brain: tumors. Eur Radiol. 1999;9(6):1047-54. doi: 10.1007/s003300050790.

24. Leite CC. Espectroscopia de prótons por ressonância magnética. Radiol Bras. 2001;34(1):V-VI. Disponível em: http://www.scielo.br/pdf/rb/v34n1/12559.pdf.

25. Burtscher IM, Holtas S. Proton magnetic resonance spectroscopy in brain tumors: clinical applications. Neuroradiology. 2001;43(5):345-52. Available from: http:// goo.gl/d7hM72.

26. Pouwels PJ, Brockmann K, Kruse B, Wilken B, Wick M, Hanefeld F, Frahm J. Regional Age Dependence of Human Brain Metabolites from Infancy to Adulthood Detected by Quantitative Localized Proton MRS. Pediatr Res. 1999;46(4):474-85 . doi:10.1203/00006450-19991000000019.

27. Srinivasan R, Phillips JJ, Vandenberg SR, Polley MY, Bourne G, Au A, Pirzkall A, Cha S, Chang SM, Nelson SJ. Ex vivo MR spectroscopic measure differentiates tumor from treatment effects in GBM. Neuro Oncol. 2010;12(11):115261. doi: 10.1093/neuonc/noq075.

28. Cheng LL, Ma MJ, Becerra L, Ptak T, Tracey I, Lackner A, González RG. Quantitative neuropathology by high resolution magic angle spinning proton magnetic resonance spectroscopy. Proc Natl Acad Sci USA. 1997;94:6408-13. Available from: http://www.pnas.org/ content/94/12/6408.long.

29. Poptani H, Gupata RK, Roy R, Pandey R, Jain VK, Chhabra DK. Characterization of intracranial Mass Lesions whith in vivo proton MR spectroscopy. AJNR Am J Neuroradiol. 1995;16:1593-603. Available from: http://www.ajnr.org/ content/16/8/1593.long.

30. Hollingworth W, Medina LS, Lenkinski RE, Shibata DK, Bernal B, Zurakowski D, Comstock B, Jarvik JG. A systematic literature review of magnetic resonance spectroscopy for the characterization of brain tumors. Am J Neuroradiol. 2006;27(7):1404-11. Available from: http:// www.ajnr.org/content/27/7/1404.long.

31. Otto D, Hennig J, Ernst T. Human brain tumors: assessment with in vivo proton MR spectroscopy. Radiology. 1993;186(3):745-52. doi: http://dx.doi.org/10.1148/ radiology.186.3.8430183.

32. Kinoshita Y, Kajiwara H, Yokota A, Koga Y. Proton magnetic resonance spectroscopy of astrocytic tumors: an in vitro study. Neurol Med Chir (Tokyo). 1993;33(6):350-9. doi: http://dx.doi.org/10.2176/nmc.33.350.

33. Carpinelli G, Carapella CM, Palombi L, Raus L, Caroli F, Podo F. Differentiation of glioblastoma multiforme from astrocytomas by in vitro $1 \mathrm{H}$ MRS analysis of human brain tumors. Anticancer Res. 1996;16(3B):1559-63.

34. Carapella CM, Carpinelli G, Knijn A, Raus L, Caroli F, Podo F. Potential role of in vitro $1 \mathrm{H}$ magnetic resonance spectroscopy in the definition of malignancy grading of human neuroepithelial brain tumours. Acta Neurochir Suppl. 1997;68:127-32.

35. Zakrzewski K, Kreisel J, Polis L, Nowosławska E, Liberski PP, Biegański T. Clinical application of proton magnetic resonance spectroscopy for differential diagnosis of pediatric posterior fossa tumors. Neurol Neurochir Pol. 2001;35(Suppl 5):19-25.

36. Kaminogo M, Ishimaru H, Morikawa M, Ochi M, Ushijima R, Tani M, Matsuo Y, Kawakubo J, Shibata S. Diagnostic potential of short echo time MR spectroscopy of gliomas with single-voxel and point-resolved spatially localised proton spectroscopy of brain. Neuroradiology. 2001;43(5):353-63.

37. Fountas KN, Kapsalaki EZ, Gotsis SD, Kapsalakis JZ, Smisson HF 3rd, Johnston KW, Robinson JS Jr, Papadakis N. In vivo proton magnetic resonance spectroscopy of brain tumors. Stereotact Funct Neurosurg. 2000;74(2):83-94. doi: $10.1159 / 000056467$.

38. Fountas KN, Kapsalaki EZ, Vogel RL, Fezoulidis I, Robinson JS, Gotsis ED. Noninvasive histologic grading of solid astrocytomas using proton magnetic resonance spectroscopy. Stereotact Funct Neurosurg. 2004;82 (2-3):90-7. doi: 10.1159/000077458.

39. Yang D, Korogi Y, Sugahara T, Kitajima M, Shigematsu Y, Liang L, Ushio Y, Takahashi M. Cerebral gliomas: prospective comparison of multivoxel 2D chemical-shift imaging proton MR spectroscopy, echoplanar perfusion and diffusion-weighted MRI. Neuroradiology. 2002;44(8):65666. doi: 10.1007/s00234-002-0816-9.

40. Möller-Hartmann W, Herminghaus S, Krings T, Marquardt G, Lanfermann H, Pilatus U, Zanella FE. Clinical application of proton magnetic resonance spectroscopy in the diagnosis of intracranial mass lesions. Neuroradiology. 2002;44(5):371-81. doi: 10.1007/s00234-001-0760-0

41. Bulakbasi N, Kocaoglu M, Ors F, Tayfun C, Uçöz T. Combination of single-voxel proton MR spectroscopy and apparent diffusion coefficient calculation in the evaluation of common brain tumors. AJNR Am J Neuroradiol. 2003;24(2):225-33. Available from: http://www.ajnr.org/ content/24/2/225.long.

42. Law M, Yang S, Wang H, Babb JS, Johnson G, Cha S, Knopp EA, Zagzag D. Glioma grading: sensitivity, specificity, and predictive values of perfusion MR imaging and proton MR spectroscopic imaging compared with conventional MR imaging. AJNR Am J Neuroradiol. 2003;24(10):1989-98. Available from: http://www.ajnr.org/content/24/10/1989. long.

43. Murphy PS, Rowland IJ, Viviers L, Brada M, Leach MO, Dzik-Jurasz AS. Could assessment of glioma methylene lipid resonance by in vivo (1)H-MRS be of clinical value? Br J Radiol. 2003;76(907):459-63.

44. Magalhaes A, Godfrey W, Shen Y, Hu J, Smith W. Proton magnetic resonance spectroscopy of brain tumors correlated with pathology. Acad Radiol. 2005;12 (1):51-7. doi: 
10.1016/j.acra.2004.10.057.

45. Chen CY, Lirng JF, Chan WP, Fang CL. Proton magnetic resonance spectroscopy-guided biopsy for cerebral glial tumors. J Formos Med Assoc. 2004;103(6):448-58.

46. Xu M, See SJ, Ng WH, Arul E, Back MF, Yeo TT, Lim CC. Comparison of magnetic resonance spectroscopy andperfusion-weighted imaging in presurgical grading of oligodendroglial tumors. Neurosurgery. 2005;56(5):919-26; discussion 919-26. doi: 10.1227/01. NEU.0000157957.67708.3E.

47. Toyooka M, Kimura H, Uematsu H, Kawamura Y, Takeuchi $\mathrm{H}$, Itoh $\mathrm{H}$. Tissue characterization of glioma by proton magnetic resonance spectroscopy and perfusion-weighted magnetic resonance imaging: glioma grading and histological correlation. Clin Imaging. 2008;32(4):251-8. doi: 10.1016/j. clinimag.2007.12.006.

48. Chawla S, Wang S, Wolf RL, Woo JH, Wang J, O'Rourke DM, Judy KD, Grady MS, Melhem ER, Poptani H. Arterial spin-labeling and MR spectroscopy in the differentiation of gliomas. AJNR Am J Neuroradiol. 2007;28(9):1683-9. Available from: http://www.ajnr.org/content/28/9/1683. long.

49. Zhou GF, Wang XY, Gong CG, Liu F, Wang RW. Value of protonmagneticresonancespectroscopywithtwodimensional chemical-shift imaging in evaluating brain gliomas. Nan Fang Yi Ke Da Xue Xue Bao. 2008;28(8):1342-4. Available from: http://www.j-smu.com/pdf2/200808/2008081342.pdf.

50. Senft C, Hattingen E, Pilatus U, Franz K, Schänzer A, Lanfermann H, Seifert V, Gasser T. Diagnostic value of proton magnetic resonance spectroscopy in the noninvasive grading of solid gliomas: comparison of maximum and mean choline values. Neurosurgery. 2009;65(5):908-13; discussion 913. doi: 10.1227/01.NEU.0000356982.82378.BA.

51. Weis J, Ring P, Olofsson T, Ortiz-Nieto F, Wikström J. Short echo time MR spectroscopy of brain tumors: grading of cerebral gliomas by correlation analysis of normalized spectral amplitudes. J Magn Reson Imaging. 2010;31(1):3945. doi: 10.1002/jmri.21991.

52. Frati A, Pichierri A, Bastianello S, Raco A, Santoro A, Esposito V, Giangaspero F, Salvati M. Frameless stereotactic cerebral biopsy: our experience in 296 cases. Stereotact Funct Neurosurg. 2011;89(4):234-45. doi: 10.1159/000325704.

53. Benedetti E, Galzio R, Laurenti G, D’Angelo B, Melchiorre E, Cifone MG, Fanelli F, Muzi P, Coletti G, Alecci M, Sotgiu A, Cerù MP, Cimini A. Lipid metabolism impairment in human gliomas: expression of peroxisomal proteins in human gliomas at different grades of malignancy. Int $\mathbf{J}$ Immunopathol Pharmacol. 2010;23(1):235-46.

54. Zeng Q, Liu H, Zhang K, Li C, Zhou G. Noninvasive evaluation of cerebral glioma grade by using multivoxel 3D proton spectroscopy. Magn Reson Imaging. 2011;29(1):2531. doi: 10.1016/j.mri.2010.07.017.

55. Zou QG, Xu HB, Liu F, Guo W, Kong XC, Wu Y. In the assessment of supratentorial glioma grade: the combined role of multivoxel proton MR spectroscopy and diffusion tensor imaging. Clin Radiol. 2011;66(10):953-60. doi: 10.1016/j.crad.2011.05.001. 